\title{
Polyethylene Bearing Wear in the Oxford Unicompartmental Knee
}

\author{
Priyanka Ghosh ${ }^{1}$, Hasan Mohammad ${ }^{2}$, Stephen Mellon², David Murray² \\ ${ }^{1}$ Indiana University School of Medicine, ${ }^{2}$ Oxford Orthopedic Engineering Centre, \\ University of Oxford
}

Background and Hypothesis: The Oxford Knee is a unicompartmental knee replacement (UKR) for patients with end-stage knee osteoarthritis (OA). It contains a polyethylene (PE) mobile bearing between the femoral and tibial components that helps maintain normal knee kinematics. Unfortunately bearing wear is one of the leading causes of UKR failure. In this study, we sought to investigate PE wear rate over time and the initial deformation (creep) in early months.

Project Methods: This study used radiostereometric analysis (RSA) to precisely track polyethylene bearing thickness over time in 40 medial Oxford UKR patients. The patients underwent stereo radiographs at 1 week post-op (reference), 3 months, 6 months, 1 year, 2 years, and 5 years. Model-based RSA software was used to determine the exact position of the femoral and tibial components, and Matlab was used to calculate the minimum linear distance between these two components, taken as bearing thickness.

Results: The average wear rate was $0.32 \mathrm{~mm} / \mathrm{yr}$ (SD 0.42) in the first 3 months, $0.16 \mathrm{~mm} / \mathrm{yr}$ (SD 0.55) from 3 to 6 months, and $0.07 \mathrm{~mm} / \mathrm{yr}$ (SD 0.03) between 1 and 5 years. No correlation was found between wear rate and BMI, fixation, implant size, or 5 year Oxford Knee Score.

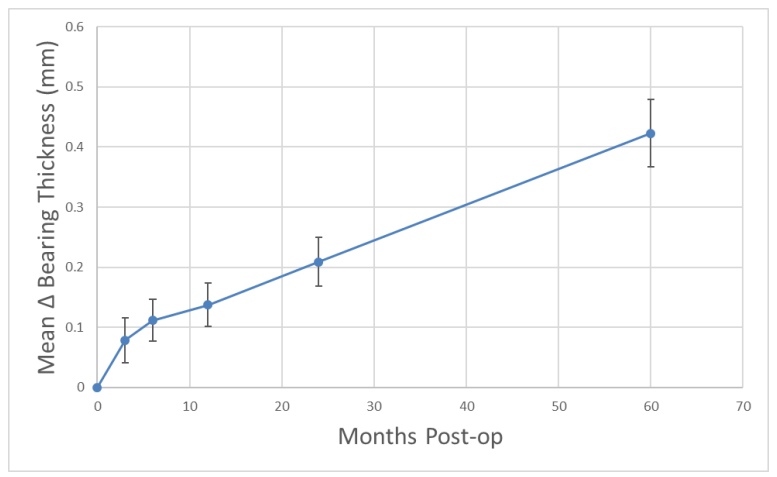

Conclusion and Potential Impact: This was the first study to investigate detailed early and late time points of polyethylene wear in the Oxford UKR. Mean bearing wear was higher in the first 6 months, presumably due to creep, and significantly reduced from 1 year onwards, presumably while true wear took place. 